Counting constituents in molecular complexes by fluorescence photon antibunching

S. Fore, T. Laurence, C. Hollars, T. Huser

April 23, 2007

IEEE Journal of Selected Topics in Quantum Electronics 
This document was prepared as an account of work sponsored by an agency of the United States Government. Neither the United States Government nor the University of California nor any of their employees, makes any warranty, express or implied, or assumes any legal liability or responsibility for the accuracy, completeness, or usefulness of any information, apparatus, product, or process disclosed, or represents that its use would not infringe privately owned rights. Reference herein to any specific commercial product, process, or service by trade name, trademark, manufacturer, or otherwise, does not necessarily constitute or imply its endorsement, recommendation, or favoring by the United States Government or the University of California. The views and opinions of authors expressed herein do not necessarily state or reflect those of the United States Government or the University of California, and shall not be used for advertising or product endorsement purposes. 


\title{
Counting constituents in molecular complexes by fluorescence photon antibunching
}

\author{
Samantha Fore, Ted A. Laurence, Christopher W. Hollars, Thomas Huser
}

\begin{abstract}
Modern single molecule fluorescence microscopy offers new, highly quantitative ways of studying the systems biology of cells while keeping the cells healthy and alive in their natural environment. In this context, a quantum optical technique, photon antibunching, has found a small niche in the continuously growing applications of single molecule techniques to small molecular complexes. Here, we review some of the most recent applications of photon antibunching in biophotonics, and we provide a guide for how to conduct photon antibunching experiments at the single molecule level by applying techniques borrowed from time-correlated single photon counting. We provide a number of new examples for applications of photon antibunching to the study of multichromophoric molecules and small molecular complexes.
\end{abstract}

Index Terms - Single Molecule Fluorescence, Photon Antibunching, Time-Correlated Single Photon Counting, Hanbury-Brown and Twiss, Molecular Complexes

\section{INTRODUCTION}

In the case of many biochemical pathways inside living cells the protein constituents are often known but the exact stochiometry of the protein-protein interactions or protein-DNA and protein-RNA interactions remains largely unknown [1] Complex functional events in cells, i.e. cell signaling [1] or DNA repair $[2,3]$, require that multiple proteins come together and form complexes - some of them potentially forming dimers or trimers in order to perform specific functions [4]. This complex problem is particularly well suited for study by single

Manuscript received March 16, 2007. S. Fore acknowledges funding from the student employee graduate fellowship program at Lawrence Livermore National Laboratory, and the Center for Biophotonics. The Center for Biophotonics, an NSF Science and Technology Center, is managed by the University of California, Davis, under Cooperative Agreement No. PHY 0120999. T. Huser also acknowledges support by the Clinical Translational Science Center under Grant Number UL1 RR024146 from the National Center for Research Resources (NCRR) a component of the National Institutes of Health (NIH), and NIH Roadmap for Medical Research. T. Laurence acknowledges support from the LLNL LDRD program. Work at LLNL was performed under the auspices of the U.S. Department of Energy by the University of California, Lawrence Livermore National Laboratory, under contract No. W-7405-Eng-48.

S. Fore and T. Huser are with the NSF Center for Biophotonics Science and Technology, and the Department of Internal Medicince, University of California, Davis, 2700 Stockton Blvd, Suite 1400, Sacramento, CA 95817 (phone: 916-734-1772; e-mail: trhuser@ucdavis.edu). T. Laurence and C. Hollars are with Lawrence Livermore National Laboratory, Livermore, CA 94550 USA, and the NSF Center for Biophotonics Science and Technology. molecule fluorescence techniques, because most of the proteins that are involved in these processes are known and can be optically labeled. The large number and complex interactions between these proteins, however, make it difficult to observe all these processes in a single experiment. It is therefore advantageous to develop novel techniques within the framework of single molecule fluorescence microscopy [5], that allow for the determination of the exact number of fluorescently labeled molecules that are present within the tightly focused laser spot of a confocal microscope. Most of these recently developed techniques, such as fluorescence correlation spectroscopy (FCS), fluorescence burst analysis, fluorescence photon antibunching, or alternating laser-excitation fluorescence resonance energy transfer, to name a few, are based on specific qualities of single fluorescent molecules and rely on sophisticated tools, such as time-correlated single photon counting (TCSPC). In this article, we specifically review recent developments in fluorescence photon antibunching and provide a practical guide for researchers that might want to implement this important technique in their own research.

Fluorescence photon antibunching exploits the fact that a single molecule can only emit one photon at a time. This means that even at optical saturation of the fluorescence excitation of a single fluorescent molecule, consecutive photons are emitted with a temporal gap that is on average of order of the excited state lifetime, i.e. typically a few nanoseconds. Only two or more independent emitters can emit photons simultaneously. This discrete emission of photons from single molecules or small molecular complexes can be exploited and investigated if we measure the time-difference between consecutively emitted photons. Such measurements are most practically performed in a Hanbury-Brown and Twiss interferometer setup [6] in which the photon flux is split in half and detected by separate photon detectors. The events from these detectors are then cross-correlated to determine the number of coincident photon pairs. Fluorescence photon antibunching was first observed in the resonance fluorescence of individual sodium atoms in atomic beams [7]. More recent experiments have extended this approach to the fluorescence of single fluorescent molecules embedded in a host matrix at low temperatures [8]. Here, this technique was effectively used to prove that indeed only fluorescence from single fluorescent molecules was observed. Shortly thereafter, this approach was expanded to the study of single molecules at room temperature [9-12]. It has since then gained significant momentum in quantum dot research to determine the quantum behavior of single fluorescent quantum dots [13-16]. The introduction of pulsed laser excitation has greatly benefited the applications of photon antibunching at the 
single molecule level $[11,17]$ and enabled the study of multiple photophysical parameters simultaneously [18]. During the last few years, this technique was also extensively used to determine the number of active emitting sites in multichromophoric molecules, ranging from large conjugated molecules $[19,20]$ to small dendritic molecules with very few chromophore units [21-27], and to determine the number of active chromophore units in fluorescent proteins [28]. Other recent applications have led to applications in photonic wire structures and macromolecular complexes [29-31].

Photon antibunching has been especially extensively used in the analysis of multichromophoric molecules, e.g. dendrimers, fluorescent proteins, and conjugated polymers. Conjugated polymers are organic semiconducting materials that can easily be processed in organic solvents [32]. In an oversimplified picture these macromolecules can be thought of as resembling organic dyes or chromophores on a string. These materials are promising candidates for novel, highly flexible electronics applications, such as large-scale candescent displays with low power consumption, or photovoltaic devices. They can be processed by simple ink-jet printing technology, making them interesting materials for small-scale prototyping or large-scale production. In a few instances, in a water-soluble form they have even found use as substrates for fluorescence enhancement of commonly used fluorophores in biodetection [33, 34]. More recent work even suggests that based on their specific three-dimensional structure and conformation, conjugated polymers appear to exhibit exciting new properties, which could turn them into highly stable and monochromatic fluorescent probes [35].

Of particular interest for potential biological applications are some even more recently discovered emission properties of conjugated polymers [35-38]. Barnes et al. used special processing to modify the conformation of individual polymer molecules. They used a microdroplet-generating nozzle to spray dilute solutions of MEH-PPV and CN-PPV into a vacuum. The concentration of the polymer was chosen such that on average there was only one or no polymer molecule contained in every microdroplet. After traveling $\sim 20 \mathrm{~cm}$ in vacuum, the microdroplets hit a glass substrate to which they adhered. These samples showed a number of remarkable properties when investigated by single molecule fluorescence spectroscopy. First, the emission spectra were extremely narrow [36]. Then, the photon emission sequence revealed a clear signature of photon antibunching, indicating that only a single chromophore unit was responsible for the emission [35]. Lastly, after transfer to air, these samples could be interrogated for up to 1 hour without showing any signs of photobleaching [38]. This is particularly remarkable as single polymer molecules are usually very sensitive to photobleaching and are virtually impossible to probe in an open-air environment. Typically, they have to be covered by a thick layer of nonfluorescent polymers to slow down oxygen diffusion in order to prevent rapid photobleaching.

\section{MATERIALS AND MethodS}

All of the experimental results shown in this paper were obtained with confocal fluorescence microscopes based on different platforms (Zeiss Axiovert 200, Nikon TE300, Olympus IX71) with sample scanning. Both home-built and commercial (PicoQuant MicroTime200) microscopes have been used in theses studies. Fluorescence excitation is achieved by routing a collimated laser beam into the microscope. Bandpass filters are used to remove plasma lines or Raman scattered light from the laser beams. A dichroic mirror reflects the laser beam into a microscope objective with high numerical aperture (NA 1.3 - NA 1.45). Fluorescence is collected by the same microscope objective and passes a longpass filter to remove any remaining laser light. The emission is split to form a Hanbury-Brown and Twiss interferometer with two avalanche photodiode detectors (SPCM-AQR-14, PerkinElmer). Shortpass or bandpass filters are placed in front of both APDs to reject near-infrared photons emitted by the APD modules. Continuous wave excitation is achieved by the use of either Argon ion lasers (Innova 90c, Coherent Inc., $488 \mathrm{~nm}, 514 \mathrm{~nm}$ lines) or Helium Neon lasers. A TimeHarp100 TCSPC card (Picoquant) is used to acquire photon arrival time histograms using one detector as the start signal and the other detector as the stop signal. For pulsed laser excitation, short-pulsed diode lasers are used at repetition rates from $1-20 \mathrm{MHz}$ (LDH, PicoQuant GmbH, 470 $\mathrm{nm}, 80 \mathrm{ps}$, and $635 \mathrm{~nm}, 80 \mathrm{ps})$. Samples are typically prepared by immobilizing single fluorophores, macromolecules with endogenous fluorescence (conjugated polymers), or oligonucleotides labeled with different fluorophores on a glass coverslip. In pulsed excitation mode, photon pulses from the APDs are detected by either TCSPC electronics (TimeHarp200, Time200 in combination with a router, or PicoHarp300, all Picoquant $\mathrm{GmbH}$ ), or a counter-timer board (PCI-6602, National Instruments).

The PicoHarp300 is functionally different from the TimeHarp200. Here, two functionally identical input channels are provided, as opposed to the different sync and start channels of the TimeHarp200 TCSPC board. Both of these channels are similar to the concept of the start channel, in that they use a constant fraction discriminator for detection. However, to achieve faster (4 ps) timing resolution, a time-to-digital converter (TDC) is used in place of the combination time-to-amplitude converter (TAC) and analog to digital converter (ADC) used in conventional TCSPC systems. The PicoHarp300 allows for acquisition in the standard histogramming mode, where the arrival times of the two channels are subtracted and sent to a histogramming unit. Additionally, it can be run in either T2 or T3 mode. The former, which was used for the antibunching measurements reported here, simply records the absolute arrival time of detected photons in both channels with 4 ps resolution. Photon antibunching histograms are then obtained by home-written software routines that determine events with coincident photon arrival times in different channels (Igor Pro, Wavemetrics), or by calculating cross-correlations between different channels [39]. 


\section{RESUlts AND DiscusSiON}

\section{A. Photon antibunching as a characteristic signature of single fluorescent molecules}

Photon antibunching in the fluorescence of single molecules is a result of the emission properties of an isolated quantum system that undergoes excitation-emission cycles. In the oversimplified picture of a single, isolated two-level system, photons can only be emitted one at a time. The distribution of the time intervals between consecutive pairs of photons depends only on the system's excited state lifetime and rate at which it is excited. The finite time period that it takes for such a quantum system to undergo an excitation-emission cycle manifests itself as a dip in the second-order intensity correlation function for short time intervals [40]

$$
g^{(2)}(t)=\frac{\left\langle I\left(t_{0}\right) I\left(t_{0}+t\right)\right\rangle}{\langle I\rangle^{2}}
$$

where $I\left(t_{0}\right)$ is the photon rate at time $t_{0}$. Recent experiments have extended this analysis to the study of single fluorescent molecules at low [8] and room temperatures [9-11, 41, 42] as well as the investigation of the quantum behavior of single semiconductor quantum dots [13, 14, 43]. More recently, our group has shown that even large multi-chromophoric molecules, such as conjugated polymers with a molecular weight of up to 1MDalton can exhibit a signature characteristic of photon antibunching, but this behavior is modulated by the polymer conformation ([19], see also Figure 2).

The second-order intensity correlation function is typically determined by conducting a "classical" Hanbury-Brown and Twiss photon correlation experiment [6], which measures the temporal separation between consecutively arriving photons. In this case the photon flux from the sample is divided equally between two single photon-counting detectors (e.g. avalanche photodiodes (APDs)). This approach is required because the response and dead times of most photodetectors are much longer than typical excited state fluorescence lifetimes. A typical setup for determining fluorescence photon antibunching at the single molecule level is depicted in Figure 1a. Fluorescence at the single molecule level is excited and detected by a confocal fluorescence microscope with high photon collection efficieny (high NA optics, detectors with high quantum efficiency). The pulses from the 2 photodetectors are used as start-stop signals for a multi-channel scaler, a TCSPC board, or photon event counters and are recorded with time-tags with picosecond to nanosecond time resolution. Once a sufficient number of photons has been detected, the photon-pair arrival time histogram will exhibit a well-expressed dip for short arrival times (see figure $2 \mathrm{~b}$ ), which reflects the fact that the probability for detecting a second photon within a very short time after the first one has been detected is very low. Signals from one of the APDs are typically delayed by an electronic delay line (e.g. a loop of BNC cable, 100 ns long) in order to enable the collection of negative coincidence times. This is the case when by chance the first ("start") photon should arrive at the detector that is configured for the detection of "stop"-photons. The time resolution of such a system is ultimately determined almost exclusively by the timing jitter of the photon detectors, which is
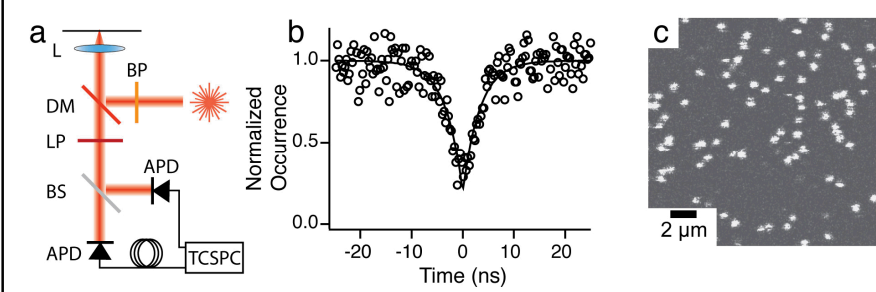

Figure 1. Photon-pair arrival time analysis of single molecule fluorescence emission. (a) The temporal separation of individual photon pairs can be analyzed by a classical Hanbury-Brown and Twiss experiment. Here, the fluorescence photon flux is split in two by a $50 / 50$ non-polarizing beamsplitter (BS) and detected by two avalanche photo diodes (APD). By delaying the arrival time of signals from one detector, coincident photon events can be detected if the delay time is known. (b) Photon-pair correlation analysis of $\sim 1000$ molecules of Rhodamine 6G probed individually by the setup shown in (a). This histogram was acquired over a time period of 10 minutes. Single fluorescent molecules can only emit one molecule at a time, which results in an anti-correlation of photon events for times shorter than the fluorescence lifetime. By fitting such a histogram, both the fluorescence lifetime and the number of molecules probed in the excitation spot can be extracted. (c) Confocal fluorescence micrograph of the sample of Rhodamine $6 \mathrm{G}$ molecules used to obtain the antibunching correlation plot shown in (b). Indivual R6G molecules were dispersed onto a glass surface by spin-coating out of a dilute ethanol solution. By slowly scanning such a surface in the confocal microscope, only one molecule is addressed at any given point in time, resulting in an optimum photon antibunching distribution.

approximately 300 ps for standard single photon-counting avalanche photodiodes, but can be as short as 50 ps with more recent versions of APDs. Another important detail of any experimental photon antibunching system is the use of shortpass filters in front of the APD detectors. APDs operated in the Geiger mode tend to emit an infrared photon for every photon that they detect. If the APDs are carefully aligned with respect to each other, these infrared photons can trigger the coincident "stop" event, even though no "real" stop event has occurred yet. The only way to remove such artificial coincidence events is by using efficient shortpass filters in front of the APDs. Furthermore, other background contributions, such as Rayleigh scattered light leaking through the longpass filters, residual substrate fluorescence, and Raman scattered light from the substrate, should be removed as much as possible. This background will also lead to an artificial increase in the number of coincident events, which leads to a higher count rate at the coincidence time, $\mathrm{t}=0$, and thus, less prominent dips in the autocorrelation at this time, as can be seen by the dip in Figure $2 \mathrm{~b}$ not extending all the way to zero coincidence events.

If the coincidence range is limited to $0-100 \mathrm{~ns}$, spatial diffusion of molecules in and out of the detection volume as well as triplet state shelving [44] can be ignored because these effects occur on time scales much longer than photon antibunching. Based on these assumptions the normalized second order intensity correlation function (Eq. (1)) [44] reduces to

$g^{(2)}(t)=\left(\frac{N(N-1)}{N^{2}}+\frac{1}{N}\left(1-e^{-|t / \tau|}\right)\right)$

where $\tau=1 /\left(k_{1}+k_{2}\right), k_{1}$ is the effective excitation pump rate, $k_{2}$ is the rate of spontaneous emission from the excited state, and $N$ the number of active emitters. This equation fits the experimentally obtained photon-pair arrival time histograms 

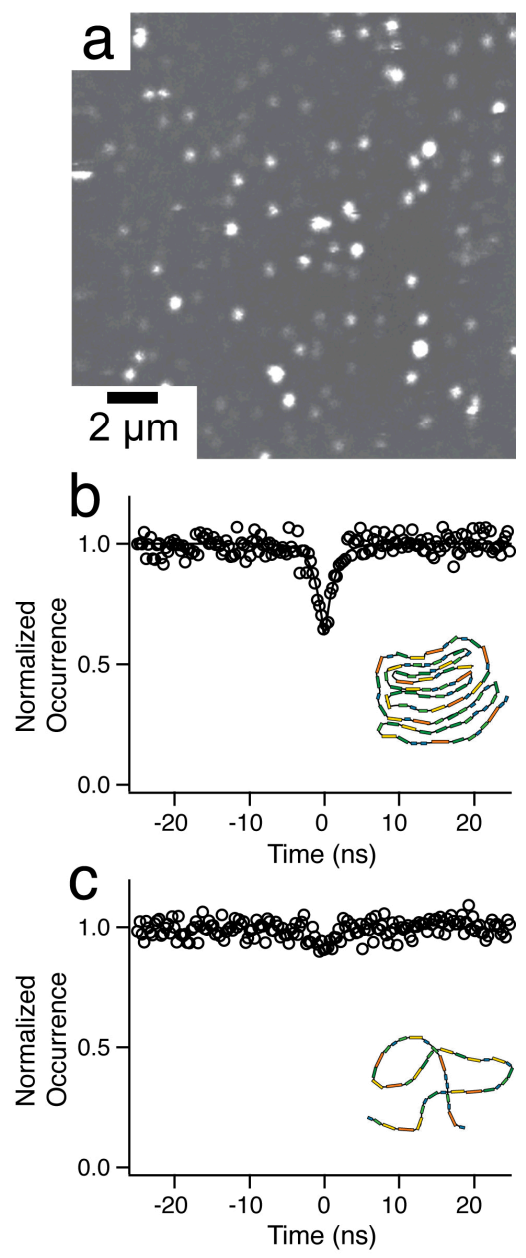

Figure 2: Fluorescence photon antibunching spectroscopy of the multichromophoric conjugated polymer MEH-PPV. These data were acquired by continuous wave excitation of MEH-PPV fluorescence and scanning across many $100 \mathrm{~s}$ of individual MEH-PPV molecules sequentially, while building up photon pair arrival time histograms. (a) 20 $\mu \mathrm{m} \times 20 \mu \mathrm{m}$ confocal fluorescence image of single MEH-PPV molecules dispersed on a glass substrate from toluene solvent. The corresponding photon-pair histogram is shown as (b). Histogram (b) was fit using eq. (2) (solid line) resulting in values of $\tau=1.3 \mathrm{~ns}, \mathrm{~N}=2.4$. (c) Photon antibunching histogram for individual MEH-PPV molecules spun from chloroform solution. Insets in (b) and (c) show rough schematics of a proposed model for the three-dimensional structure of MEH-PPV in each solvent, respectively.

very well and can be used to extract information about the system under investigation, i.e. the excited state lifetime $(\tau)$ and the number of active molecules $(N)$ within the confocal excitation spot.

\section{B. Photon antibunching with continuous wave excitation}

The validity of the experimental Hanbury-Brown and Twiss setup for photon antibunching measurements can be demonstrated by conducting a photon-pair coincidence analysis on single molecules of a well-characterized system, i.e. the laser dye rhodamine 6G (R6G). Figure 1c shows a typical scanning confocal fluorescence micrograph of R6G molecules spincast on a carefully cleaned glass substrate at $10^{-10} \mathrm{M}$ concentration. In this image, each bright spot corresponds to the fluorescence emission of a single R6G molecule. The corresponding plot in Figure $1 \mathrm{~b}$ shows the normalized correlated photon pair distribution histogram generated by scanning a $100 \mu \mathrm{m}$ x 100

$\mu \mathrm{m}$ area of the sample with an excitation power density of 1 $\mathrm{kW} / \mathrm{cm}^{2}$, thereby addressing $\sim 1000$ molecules one by one in series, and calculating a histogram of all photon events for the entire time period that it takes to conduct the $\mathrm{x}-\mathrm{y}$ scan (typically about 10 minutes). The resulting histogram data for the sample in Figure 1c are shown in Figure $1 \mathrm{~b}$ with a time bin of 288 ps. This histogram is the result of the 5 consecutive image scan such as the one shown in Figure 1c, where each image scan was conducted on a new, previously un-scanned area.

By using equation (2), a least squares fit of the coincidence histogram for the R6G sample results in values of $\tau=3.3 \mathrm{~ns}$ and $\mathrm{N}=1.2$. In this experiment, a time-correlated single photon counting (TCSPC) board with 36 ps time-resolution was used to obtain the histograms rather than a multi-channel scaler. After deconvolution with the instrument response function and fluorescence lifetime, the result for the excited state lifetime of R6G fits well with literature lifetimes of 3.65 ns $[9,45]$. Of special note is the fact that the additional fit parameter in equation (2), $N$, provides a quantitative measure for the average number of independent emitters simultaneously interrogated within the diffraction-limited focused excitation spot. Given the low concentration of R6G in this analysis, which is represented by the high average distance between individual fluorescence spots in Figure 1c, the focused beam of the microscope is primarily probing single R6G molecules. This is reflected in a fit value of $N=1.2$. As discussed above, the deviation of this value from unity is attributed to background from Raman scattering and spurious fluorescence in the glass substrate.

We have recently also used the Hanbury-Brown and Twiss experiment to study the photophysical effects of different chain conformations of multichromophoric conjugated polymers on their emission [19]. For our study, single molecules of the conjugated polymer MEH-PPV were spincast onto glass substrates from solutions with different solvent polarity. It has been shown by dynamic light scattering that MEH-PPV in the non-polar solvent toluene forms tight coils with diameters of about $10 \mathrm{~nm}$, while MEH-PPV in the polar solvent chloroform forms extended coils ("relaxed" structures) with a diameter of about $20 \mathrm{~nm}$ [46]. Figure 2a shows a typical scanning confocal fluorescence micrograph of single MEH-PPV molecules dispersed from toluene solution. The corresponding photon pair distribution histogram for toluene is plotted in Figure 2b. Here, every polymer molecule was probed within a total interrogation time of less than $100 \mathrm{~ms}$ to avoid adverse effects from photobleaching. As can be seen from the histogram in Figure $2 b$ the photoluminescence of isolated molecules of MEH-PPV spin-cast from toluene reveals photon antibunching as indicated by the dip in the photon pair arrival time histogram. The fact that at time $t=0$ the dip in the histogram does not extend to zero coincidence counts indicates that on average the collapsed-chain MEH-PPV molecules that result from processing in toluene seem to have more than one active emission center. This value can be extracted by fitting the correlation histogram with equation (2), resulting in a value of $\mathrm{N}=2.4$. As pointed out in [19] this result is quite remarkable, because it indicates that polymer chains that consist of hundreds of chromophore units have on average only 2 - 3 emissive sites in the collapsed chain conformation. All other chromophores must therefore serve as 
efficient light-harvesting and energy transfer-sites. In contrast, MEH-PPV molecules spun-cast from chloroform solution do not exhibit a well-expressed dip in their antibunching histogram, indicating that they contain many emissive centers.

Based on these and other, spectroscopic results, we have proposed a model for the 3D structure of MEH-PPV molecules in each solvent, where MEH-PPV in toluene adopts a tight, highly folded structure that permits efficient intra-molecular energy transfer, while MEH-PPV in chloroform adopts a more open conformation leading to the emission from many chromophore units [47, 48]. Schematics of these proposed structures are shown as insets to the histograms in Figures $2 b$ and $2 \mathrm{c}$.

These experiments and all of the earlier photon antibunching experiments were conducted with continuous wave (CW) excitation, but this scheme has severe limitations. As shown above, this scheme clearly only works well if the fluorescence from many individual molecules is added up sequentially by addressing one molecule at a time. This means that photon antibunching experiments using $\mathrm{CW}$ excitation from single fluorescent molecules can really only be achieved at low temperature, where photobleaching is significantly suppressed [8] or if bleaching-resistant fluorophore, such as quantum dots [13] are used. At room-temperature a single molecule by itself does not provide enough photons in order to generate a histogram with sufficient signal-to-noise because it will ultimately photobleach within a few seconds. The main limitation here is due to the fact that for molecules excited with a continuous-wave (cw) laser beam fluorescence photons are generated at random times and we have no a-priori knowledge of when photon emission will occur. Since the reset time of the APDs is approximately $35 \mathrm{~ns}$, this causes a loss of the photons that are generated during this dead time of the detectors. Fluorescent dye molecules produce a finite number of photons before photobleaching occurs, typically $\sim 10^{6}$. With most of them wasted during the APD dead time, it is impossible to collect a sufficient number of coincident photons from a single molecule to accurately measure $\mathrm{g}^{(2)}(\tau)$, thus the need for averaging over 1000's of molecules.

Let us reconsider the main objective of any photon antibunching experiment, i.e. the detection of coincidence events, which indicate the presence of more than one emitter. With current photon-counting detector technology we have to resort to using 2 independent detectors in order to detect 2 coincident photons, because of the detector-intrinsic deadtime of typically about $35 \mathrm{~ns}$ (a single detector operating in the Geiger mode cannot detect two coincident photons). This means, we have to rely on the Hanbury-Brown and Twiss arrangement for all photon antibunching experiments. This geometry, however, already limits the chances for detecting coincident photons to the case where both photons are clearly split into two separate detection channels. If both photons went to the same detector they would not get registered as a coincidence event. This implies that our intrinsic efficiency of detecting coincident photons is only $33 \%$ to begin with, because the possible events are distributed as follows: both photons go to detector 1 (33\% probability), both photons go to detector 2 (33\% probability), or one photon goes to detector 1 and the other one goes to detector 2 (33\% probability). It should be noted, however, that this probability changes and actually goes up in favor of the two-detector arrangement for the case of more than 2 independently emitting molecules.

In CW excitation mode with TCSPC electronics, we rely on one detection channel to be defined as the "start" channel and the other one to be the "stop" channel. This arrangement artificially lowers the probability of detecting coincident photons even more, because we now arbitrarily define the channels instead of using any one of them as the "start" or "stop" channel. A "start" photon going to the "stop" channel will not get registered and is lost. Also, in CW mode we have no control over when to expect photons to arrive at the detector, thus a "start" photon will keep the electronics active until a "stop" photon has arrived ignoring any further photons going to the "start" channel until the electronics is reset. Lastly, once a photon pair has been registered, the electronics needs time to process this event, which further leads to a loss of detected photons. Somewhat of a work-around for these problems is to delay the signals from the "stop" channel by a few hundred nanoseconds (through the use of appropriate BNC cable lengths as shown in Figure 1a or other means). In this case, photons going to the "stop" channel can be detected even if they were registered a few nanoseconds before the "start" event, because their physical arrival time at the electronics is delayed.

This leads to the registration of "negative" photon pairs in CW mode - photon pairs where the arrival time is less than the delay time between the channels. The loss of photon events due to random emission times, having to define channels, and the rapid photobleaching of dyes due to continuous exposure, however, cannot be avoided.

\section{Photon antibunching using pulsed fluorescence excitation}

When photon antibunching experiments are conducted in pulsed laser excitation mode, the $\mathrm{CW}$ laser excitation is replaced by a pulsed laser with picosecond pulse width [17]. If the laser pulses are short enough (less than $100 \mathrm{ps}$ ), the probability that a single molecule emits a photon and is then immediately re-excited by the same laser pulse is very low. Thus, if we excite all molecules within the laser focus at pre-defined points in time, we know exactly when to expect fluorescence photons from these molecules. By selecting a repetition rate that is longer than the APD dead time, no photons are lost due to this downtime. Also, rapid photobleaching is alleviated because the molecules undergo much fewer excitation-emission events. This mode does, however, not help with the definition of channels if TCSPC electronics are used, thus leading to some loss of photon events if the "stop" photon was emitted to the same channel as the "start" photon. Delaying the arrival of the "stop" channel photon events, however, does still help in detecting photons that were recorded in this channel even though they did indeed arrive earlier than the "start" photon 

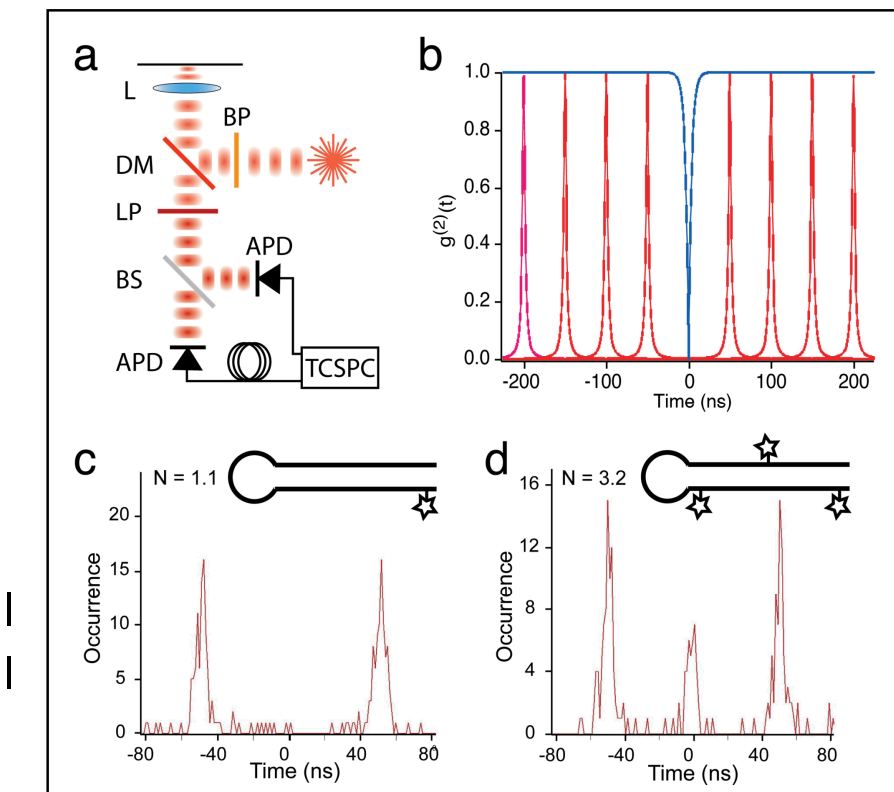

Figure 3. Fluorescence photon antibunching experiment with pulsed laser excitation. (a) A short-pulsed laser (pulse length much shorter than the fluorescence lifetime) is first sent through a bandpass (BP) filter and then coupled into a confocal microscope. The collected fluorescence photons are split in two separate detection channels as shown before in the case of $\mathrm{CW}$ excitation. (b) Calculated photon pair arrival time histogram for a single fluorescent molecule excited at $20 \mathrm{MHz}$ repetition rate. The molecule is now excited at well-defined points in time reflecting the laser repetition rate rather than randomly distributed excitation events. This results in peaks in the photon pair arrival time histogram. In contrast, the curve obtained by $\mathrm{CW}$ excitation is also shown. If single molecules are addressed, the peak at time zero (defined by the delay length) is suppressed. Representative fluorescence photon antibunching histograms for single DNA hairpin molecules labeled with (c) 1 or (d) 3 fluorescent dyes. The signal integration time for each of the histograms was approximately $5 \mathrm{~s}$ and reflects signals from just a single DNA molecule.

. The pulsed fluorescence excitation mode makes it possible to perform photon antibunching measurements on single fluorescent dye molecules without the need to average over $100 \mathrm{~s}$ of molecules. This mode of pulsed excitation of coincident photon events from individual molecules was first demonstrated by W.E. Moerner's group at Stanford University who thereby effectively created a source of single photons on demand [17] Later, Sauer et al. demonstrated the potential of this technique to simultaneously obtain a wide range of photophysical parameters [11].

This pulsed mode of fluorescence excitation is schematically represented in Figure 3a. As before the collected fluorescence photons are split in two separate detection channels. Figure $3 \mathrm{~b}$ shows the expected photon pair arrival time histogram calculated for a single fluorescent molecule that is excited at a repetition rate of $20 \mathrm{MHz}$. The molecule is now excited at well-defined points in time reflecting the laser repetition rate rather than randomly distributed excitation events. This results in peaks in the photon pair arrival time histogram.

If pulsed excitation is employed, the expression for $g^{(2)}(\tau)$ can now no longer be used to determine the values of the number of active emitters, $N$, and their fluorescence lifetime, $\tau$. In this case, however, the ratio of the area of the discrete peaks that are resulting from pulsed excitation is instrumental in determining
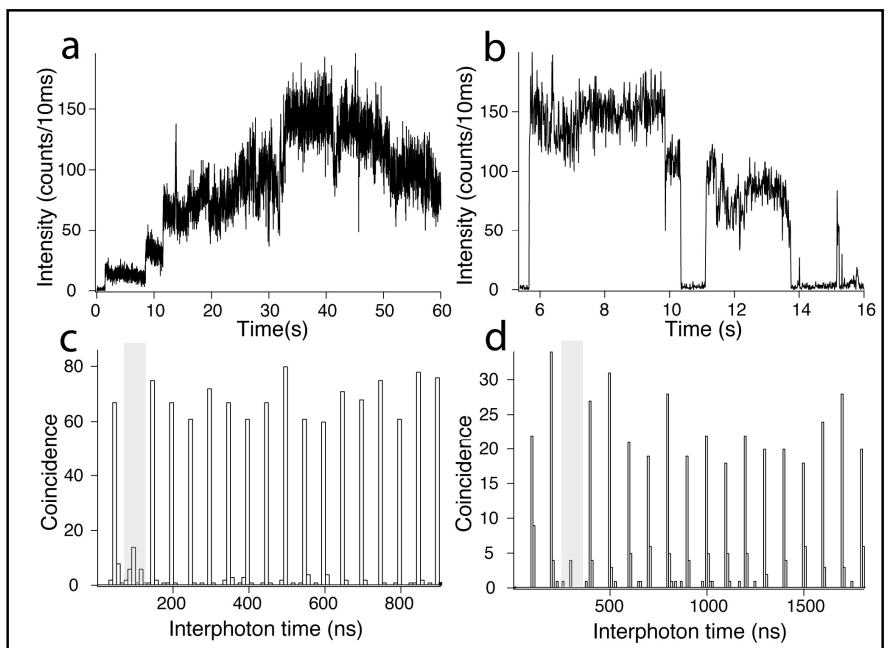

Figure 4: Spectrally selective photon antibunching experiment conducted by pulsed laser excitation of individual molecules of the conjugated polymer MEH-PPV. (a)_Intensity transient obtained at $470 \mathrm{~nm}$ excitation and signal collection through a bandpass filter with a passband from 500 $\mathrm{nm}-540 \mathrm{~nm}$. (c) Corresponding photon-pair arrival time histogram acquired over the entire 60 s time window shown in (a). (b) Intensity transient obtained from another MEH-PPV molecule at $470 \mathrm{~nm}$ excitation and signal collection through a bandpass filter with a passband from 565 $\mathrm{nm}-635 \mathrm{~nm}$. (d) Corresponding photon-pair arrival time histogram acquired over the approx. 10s time period shown in (b). Note that the laser repletion rate in (a) and (c) was $20 \mathrm{MHz}$, while in (b) and (d) it was 10 $\mathrm{MHz}$

$N$. The area of the central peak at time zero, $\mathrm{m}_{\mathrm{c}}$, with respect to the average area of all the other lateral peaks, $\bar{m}_{l}$, is now a measure of the probability of detecting photon pairs per laser pulse [11]. Specifically, $N$, the number of emitters can be determined using the following, simple expression:

$$
\frac{m_{c}}{\bar{m}_{l}}=1-\frac{1}{N}
$$

After modifying and optimizing our setup we recently demonstrated photon antibunching on single fluorescent molecules $[30,39]$. We used a simple model system - DNA hairpin molecules labeled with between 1-3 organic fluorophores to determine the accuracy with which small numbers of fluorophores can be measured [30]. In these experiments, a $640 \mathrm{~nm}$ pulsed diode laser ( 80 ps pulse width, 20 $\mathrm{MHz}$ repetition rate) was used for the excitation of discrete numbers of dye molecules attached to DNA hairpin molecules. Coincidence histograms for two samples, DNA hairpins labeled with one and three Atto655 dye molecules, respectively, are shown in Figure $3 \mathrm{c}$ and $3 \mathrm{~d}$. The histograms in Figure 3 clearly show that the inter-photon time distances are determined by the repetition rate of the laser within the accuracy of the fluorescence emission lifetime. In this case, the signal integration time for each of the histograms could be reduced to approximately $5 \mathrm{~s}$ and each histogram reflects signals from just a single DNA molecule with a discrete number of fluorophores. In addition, the absence of the peak at zero time delay as observed in Figures $3 \mathrm{c}$ and $3 \mathrm{~d}$ indicates photon antibunching, as only one photon can be emitted within a few nanoseconds after successful excitation of the molecule. If we use equation (3) to determine the number of active emitters for each of the histograms in Figure 3, we obtain, as expected, $N=1$ for the histogram acquired on the singly labeled DNA hairpin molecule and $N=3$ for the DNA molecule labeled with 3 fluorophores. 
More recently, we have revisited MEH-PPV and obtained photon antibunching histograms on individual polymer molecules. The result was even more surprising than our initial results obtained by $\mathrm{CW}$ excitation (Figure 2). These samples were processed in the traditional way - spincast from $10^{-11} \mathrm{M}$ toluene solution (unfavorable solvent, leading to partial collapse of the polymer chains) and covered by an approx.100 nm thick layer of a nonfluorescent polymer (PVB). In contrast to conducting a photon antibunching experiment on the entire emission range of MEH-PPV, we now used bandpass filters to determine spectrally-selective photon antibunching. Figure $4 \mathrm{a}$ shows an intensity transient for a collapsed-chain MEH-PPV molecule detected through a $500-540 \mathrm{~nm}$ bandpass filter. Only photons from this wavelength range were allowed to form a photon-pair histogram by sending them to 2 APDs. The result is shown in Figure 4c. Similarly, the column (Figure 4 b, d) on the right side of Figure 4 shows an intensity transient and photon pair arrival histogram of MEH-PPV when detected through a $565-635 \mathrm{~nm}$ bandpass filter. The most remarkable feature in the histograms is that with this optimized setup, individual molecules of MEH-PPV exhibited the signature of a single chromophore, i.e. the antibunching histogram stretched all the way to zero (see figure $4 \mathrm{c}$ and $4 \mathrm{~d}$, the suppressed antibunched peaks are highlighted by a gray bar). This result is significantly different from our previous result on the same system that indicated the presence of 2-3 active chromophores. At the same time, the emission transients of the MEH-PPV molecules show significant complementary trends. For the bandpass filter in the green part of the spectrum, a step-wise increase in emission intensity can be observed (see Figure 4a), while the emission to the red channel shows a step-wise decrease in intensity. This effect can be explained with the continuous blue-shift of collased-chain MEH-PPV, where first the chromophore unit with the lowest energy is excited and active, and after photobleaching of this unit, the emission shifts progressively to bluer, more highly energetic emissive states [47, 48]. This effect leads to the also observed step-wise decrease of red fluorescence emission as can be seen in Figure 4d. In addition to obtaining antibunching results on individual polymers, it should also be noted that we are now also able to obtain histograms of the distributions of active chromophore units from hundreds of individual molecules, which can be used to form antibunching distributions or distributions of the number of active emitters for many similar molecules.

The final disadvantage of having to define start and stop channels with TCSPC electronics can be eliminated if pulsed excitation is combined with routed TCSPC electronics. In this case, every photon leading to a photoelectron is registered and recorded with its macroscopic and microscopic arrival time and the channel number in which it was recorded. The difference to the previous case is that both detectors now act as "start" channels, while the reference signal from the laser acts as "stop" signal. This allows the simultaneous recording of macroscopic photon events with nanosecond time resolution, the recording of microscopic photon events with picosecond time resolution, and the recording of the channel number. In this case the main purpose of delaying the arrival of photon events in one of the two channels is to prevent the electronics from missing coincident events, because the router continuously switches

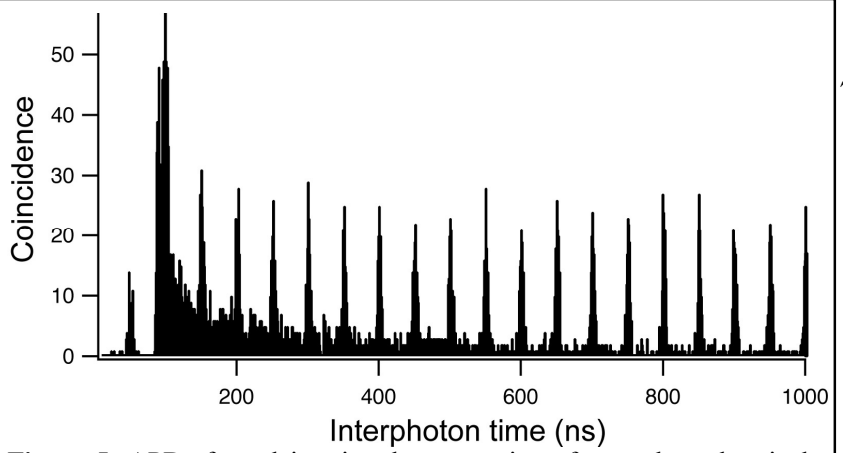

Figure 5: APD afterpulsing, i.e. the generation of secondary electrical pulses by APD detectors operating in Geiger mode can lead to erroneous coincidence events. This is shown for the example of a photon pair arrival time histogram obtained from a single MEH-PPV molecule, where the cable delay between the two detection channels was removed. The effect of APD afterpulsing leads to an exponentially decaying background contribution that peaks at approximately $90 \mathrm{~ns}$ after the detection of a photon.

through all input channels and by detecting one event in one channel it could miss a coincident event in the other channel. This mode of operation also has the advantage that now, many different photophysical parameters, e.g. intensity transients, fluorescence lifetime, number of active emitter sites, etc. can be obtained simultaneously as was first demonstrated by Weston et al [18]. This mode of operation makes photon antibunching experiments so efficient that based on the overall brightness and robustness of the probe, transients of all these photophysical parameters can be calculated and plotted.

A potential problem leading to artifacts in pulsed excitation photon antibunching detection is based on another intrinsic effect of avalanche photodiodes. Most APD detectors have a high likelihood that after the detection of a photon and the subsequent generation of a TTL output pulse, another TTL pulse will be generated without the detection of another photon. This effect, called APD afterpulsing, can be demonstrated by subtracting subsequent photons from previous photons in a continuous photon record without taking into account the channel number, i.e. whether or not both pulses arrived from the same detector, or the order in which the photons making up the pair arrived. An APD afterpulse is erroneously detected as a photon event by the TCSPC electronics. Since afterpulsing is an intrinsic product of photon detection by APDs, photon pairs that are tagged as coming from the same detector will include events in which the first event was an actual detected photon and the second is the subsequent APD afterpulse. There exists a finite probability of APD afterpulsing for a short time after the detection of a photon, and this probability decays exponentially. Figure 5 shows that the probability for detecting afterpulsing events peaks at about 90 ns after photon detection and decays exponentially. The data shown in Figure 5 are obtained from the photon emission of a single MEH-PPV molecule, excited at 20 $\mathrm{MHz}$ repetition rate. As indicated earlier, APD afterpulsing decays exponentially with time, which can be seen as an exponentially decaying coincidence background superimposed on the regularly spaced peaks in Figure 5. Here, the delay between the APD channels was removed, which very clearly emphasizes the effect of APD afterpulsing. This however, also shows, that afterpulsing can be effectively eliminated by employing proper cable delays and using photon-pairs detected from different APD's. 
An even more efficient photon detection can be obtained if instead of TCSPC electronics, photon event counters are used. Depending on the manufacturer and the frequency of an external master clock, these event counters can have time resolutions of 12.5 ns (i.e. PCI-6602, National Instruments), or even 4 ps (e.g. PicoHarp300, Picoquant $\mathrm{GmbH}$ ), where every photon event is recorded with this absolute precision in the arrival time. This last configuration is demonstrated in Figure 6 for the case of two fluorescently labeled DNA hairpin molecules. The DNA hairpin used to obtain the transients shown on the left hand side of Figure 6, Figure 6a-c, is labeled with 2 Atto655 fluorophores, where both fluorophores are separated by 11 base pairs. The DNA hairpin molecule shown on the right hand side of Figure 6, leading to the transients shown in Figure 6d-f, on the other hand, is labeled with 3 fluorophores, with inter-fluorophore distances of 11 and 12 base pairs, respectively. The antibunched peak in the histograms lies at a temporal position of $300 \mathrm{~ns}$ due to a 300 ns cable delay that was used between the two detector channel inputs on the PicoHarp300. As can be seen from the ratio of peak areas $\mathrm{N}_{\mathrm{ab}} / \mathrm{N}_{\mathrm{L}}$ as shown in Figures $6 \mathrm{a}$ and $6 \mathrm{~b}$ for the double labeled sample, the sample initially exhibits emission from both fluorophore, but within the first 10 seconds, one of the two fluorophores photobleaches, and only one active fluorophore remains for the rest of the transient. This leads to a misleading average number of active sites of $N=1$ if the photon antibunching histogram is averaged over the entire duration of the signal acquisition. The situation gets somewhat more complicated, if we consider the case of the hairpin molecule labeled with 3 fluorophores. Here, the intensity time trace and the ratio of peak areas $\mathrm{N}_{\mathrm{ab}} / \mathrm{N}_{\mathrm{L}}$ is shown in Figures $6 \mathrm{~d}$ and $6 \mathrm{e}$. In the case of no dye-dye interactions or fluorescence quenching by DNA, the intensity levels obtained from this system should correspond to three, two, or one active dye, and hence, a ratio of $0.67,0.5$, and 0 , respectively, is expected. Here, however, it appears as if the dyes on the DNA hairpin might undergo interactions with the DNA and the dyes themselves, resulting in fluctuating values for the ratio and the emission intensity, but do not provide the expected results for non-interacting independent dyes. In this case, the photon pair arrival time histogram as shown in Figure $6 \mathrm{f}$ would actually indicate an average number of 2 active fluorophores.

Finally, we would like to emphasize the importance of the right software algorithms for calculating photon antibunching histograms. Most of the histograms obtained for pulsed excitation and displayed in Figures 4-6 were generated by an algorithm that reads a photon event and tries to find a coincident event in the second channel generated at a time that is determined by the fixed cable delay between the channels. Counting a few events surrounding the time defined by the cable delay results in the generation of the non-antibunched side peaks, the area of which is used to calculate the absolute number of active emitters. Here, cross-correlation algorithms are clearly much better suited to capture every possible event and maximize the signal-to-noise ratio for the calculation of the number of active emitters. The main disadvantage of classical cross-correlation algorithms, however, is that these can be very time-consuming. Other, more time-efficient techniques can improve this problem significantly. This is demonstrated in the antibunching histogram shown in Figure $7 \mathrm{~b}$, which was

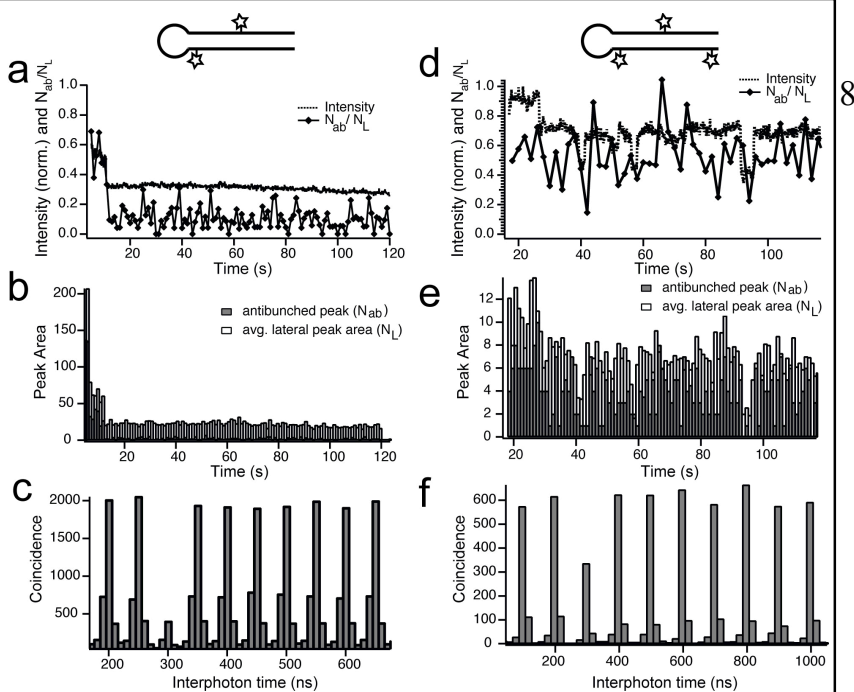

Figure 6: Photon antibunching experiments conducted on single DNA hairpin molecule labeled with 2 (left column, a-c) and 3 fluorophores (right column, d-f) with a 4-channel router and TCSPC electronics. (a, d) Time traces of normalized intensity (dotted line) and ratio of the peak area of the antibunched peak $\left(\mathrm{N}_{\mathrm{ab}}\right)$ relative to the average area of the lateral peaks $\left(\mathrm{N}_{\mathrm{L}}\right.$, solid line plus diamonds) reflecting the relative number of active fluorophores. The ratio was calculated for 1-second time bins, respectively. Discrete emission levels corresponding to three, two, or one active chromophore should result in a $\mathrm{N}_{\mathrm{ab}} / \mathrm{N}_{\mathrm{L}}$ ratio of 0.67 , 0.5 , and 0 , respectively. (b, e) Bar graphs representing the actual peak area values obtained for each one second interval and used to calculate the ratio shown in (a, and d). (c, f) Total antibunching histogram of all photons recorded over the entire acquisition time periods shown in the upper graphs.

calculated by cross-correlating the events generated during the image scan shown in Figure 7a. The specific algorithm used to calculate the histogram shown in Figure 7b, allows for arbitrary bin sizes, making cross-correlation calculations very flexible over an adjustable, wide range of correlation times. Details about this novel algorithm can be found in [39].

\section{CONCLUSIONS}

In conclusion, we have reviewed the development of photon antibunching techniques in single molecule fluorescence microscopy. Such techniques offer the promised to become effective tools to count small numbers of identical fluorophores that are used to fluorescently tag proteins or DNA, and to determine local fluctuations in the number of these molecules based on interactions between the macromolecules, even with temporal resolution. Photon antibunching can also be used to determine the inter-molecular mechanisms of energy transfer or simply the number of active sites within a multichromophoric molecule. Pulsed excitation generates photons on demand, enabling photon antibunching to be performed on single, isolated molecules. This mode also affords the possibility to determine transients of the number of active fluorescent emitters with a bin width below 1 second. We demonstrated these attributes of photon antibunching based on samples with well-defined, discrete numbers of fluorophores, as well as on large multichromophoric molecules, where the number of active emitters depends dramatically on the overall conformation of the molecule.

\section{REFERENCES}



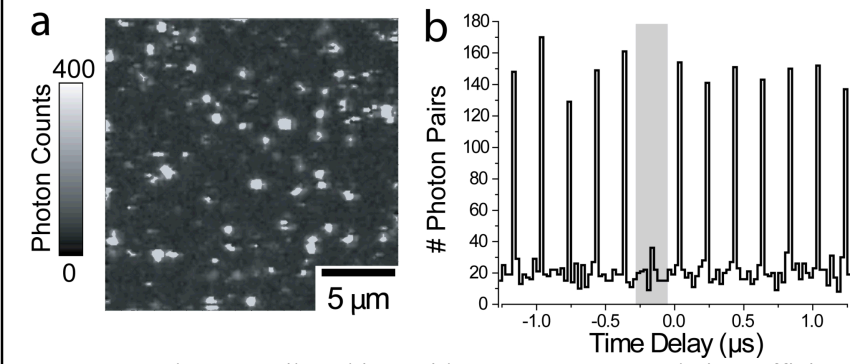

Figure 7: Photon antibunching with event counter and time-efficient cross-correlation algorithm. (a) Confocal micrograph of single Atto655 fluorophores on a glass substrate. (b) Photon antibunching histogram calculated by cross-correlating all photon events obtained during the image scan in (a). The suppressed antibunching peak indicating single molecule events is highlight by the gray bar near the center of the figure.

1. Alberts, B., et al., Molecular Biology of the Cell. 4 ed. 2002, New York: Garland Science.

2. $\quad$ Costa, M.A., et al., The eukaryotic nucleotide excision repair pathway. Biochemie, 2003. 85: p. 1083-1099.

3. Lindahl, T. and R.D. Wood, Quality control by DNA repair. Science, 1999. 286: p. 1897-1905.

4. Yang, Z., et al., Dimerization of human XPA and formation of $X P A 2-R P A$ protein complex. Biochemistry, 2002. 41: p. 13012-13020.

5. Weiss, S., Fluorescence spectroscopy of single biomolecules. Science, 1999. 283(5408): p. 1676-1683.

6. Hanbury-Brown, R. and R.Q. Twiss, Correlation between photons in two coherent beams of light. Nature (London), 1956. 177: p. 27-29.

7. Kimble, H.J., M. Dagenais, and L. Mandel, PHOTON ANTI-BUNCHING IN RESONANCE FLUORESCENCE. Physical Review Letters, 1977. 39(11): p. 691-695.

8. Basche, T., et al., Photon Antibunching in the Fluorescence of a Single Dye Molecule Trapped in a Solid. Physical Review Letters, 1992. 69(10): p. 1516-1519.

9. Ambrose, W.P., et al., Fluorescence photon antibunching from single molecules on a surface. Chemical Physics Letters, 1997. 269(3-4): p. 365-370.

10. Fleury, L., et al., Nonclassical photon statistics in single-molecule fluorescence at room temperature. Physical Review Letters, 2000. 84(6): p. 1148-1151.

11. Tinnefeld, P., C. Muller, and M. Sauer, Time-varying photon probability distribution of individual molecules at room temperature. Chemical Physics Letters, 2001. 345(3-4): p. 252-258.

12. Berglund, A.J., A.C. Doherty, and H. Mabuchi, Photon statistics and dynamics of fluorescence resonance energy transfer. Physical Review Letters, 2002. 89(6): p. 4.

13. Michler, P., et al., Quantum correlation among photons from a single quantum dot at room temperature. Nature, 2000. 406(6799): p. 968-970.

14. Lounis, B., et al., Photon antibunching in single CdSe/ZnS quantum dot fluorescence. Chemical Physics Letters, 2000. 329(5-6): p. 399-404.

15. Biebricher, A., M. Sauer, and P. Tinnefeld, Radiative and nonradiative rate fluctuations of single colloidal semiconductor nanocrystals. Journal of Physical Chemistry B, 2006. 110(11): p. 5174-5178.

16. Zinoni, C., et al., Time-resolved and antibunching experiments on single quantum dots at $1300 \mathrm{~nm}$. Applied Physics Letters, 2006. 88(13): p. 3.

17. Lounis, B. and W.E. Moerner, Single photons on demand from a single molecule at room temperature. Nature, 2000. 407(6803): $\mathrm{p}$. 491-493.

18. Weston, K.D., et al., Measuring the number of independent emitters in single-molecule fluorescence images and trajectories using coincident photons. Analytical Chemistry, 2002. 74(20): p. $5342-5349$.

19. Hollars, C.W., S.M. Lane, and T. Huser, Controlled non-classical photon emission from single conjugated polymer molecules. Chemical Physics Letters, 2003. 370(3-4): p. 393-398.

20. Bussian, D.A., et al., Photon pair correlation spectroscopy of single tetrahedraloligophenylenevinylene molecules at room temperature. Chemical Physics Letters, 2004. 388(1-3): p. 181-185.

21. Tinnefeld, P., et al., Antibunching in the emission of a single tetrachromophoric dendritic system. Journal of the American Chemical Society, 2002. 124(48): p. 14310-14311.
Hubner, C.G., et al., Photon antibunching and collective effects in the fluorescence of single bichromophoric molecules. Physical Review Letters, 2003. 91(9): p. 4.

23. Hofkens, J., et al., Revealing competitive Forster-type resonance energy-transfer pathways in single bichromophoric molecules. Proceedings of the National Academy of Sciences of the United States of America, 2003. 100(23): p. 13146-13151.

24. Bell, T.D.M., et al., Single photon emission from a dendrimer containing eight perylene diimide chromophores. Australian Journal of Chemistry, 2004. 57(12): p. 1169-1173.

25. Masuo, S., et al., Multichromophoric dendrimers as single-photon sources: A single-molecule study. Journal of Physical Chemistry B, 2004. 108(43): p. 16686-16696.

Tinnefeld, P., et al., Higher-excited-state photophysical pathways in multichromophoric systems revealed by single-molecule fluorescence spectroscopy. Chemphyschem, 2004. 5(11): p. 1786-1790.

27

Vosch, T., et al., Probing Forster type energy pathways in a first generation rigid dendrimer bearing two perylene imide chromophores. Journal of Physical Chemistry A, 2003. 107(36): p. 6920-6931.

28. Sanchez-Mosteiro, G., et al., Photon antibunching proves emission from a single subunit in the autofluorescent protein DsRed. Chemphyschem, 2004. 5(11): p. 1782-1785.

Heilemann, M., et al., Dissecting and reducing the heterogeneity of excited-state energy transport in DNA-Based photonic wires. Journal of the American Chemical Society, 2006. 128(51): p. 16864-16875. Fore, S., et al., Distribution analysis of the photon correlation spectroscopy of discrete numbers of dye molecules conjugated to DNA. Ieee Journal of Selected Topics in Quantum Electronics, 2005. 11(4): p. 873-880.

31. Widengren, J., et al., Single-molecule detection and identification of multiple species by multiparameter fluorescence detection. Analytical Chemistry, 2006. 78(6): p. 2039-2050.

32. Friend, R.H., et al., Electroluminescence in conjugated polymers. Nature, 1999. 397(6715): p. 121-128.

33. Chen, L.H., et al., Highly sensitive biological and chemical sensors based on reversible fluorescence quenching in a conjugated polymer. Proceedings of the National Academy of Sciences of the United States of America, 1999. 96(22): p. 12287-12292. Gaylord, B.S., A.J. Heeger, and G.C. Bazan, DNA detection using water-soluble conjugated polymers and peptide nucleic acid probes. Proceedings of the National Academy of Sciences of the United States of America, 2002. 99(17): p. 10954-10957.

Kumar, P., et al., Photon antibunching from oriented semiconducting polymer nanostructures. Journal of the American Chemical Society, 2004. 126(11): p. 3376-3377.

36

Kumar, P., et al., Narrow-bandwidth spontaneous luminescence from oriented semiconducting polymer nanostructures. Journal of Physical Chemistry B, 2003. 107(26): p. 6252-6257.

37. Mehta, A., et al., Oriented nanostructures from single molecules of a semiconducting polymer: Polarization evidence for highly aligned intramolecular geometries. Nano Letters, 2003. 3(5): p. 603-607. Lee, T.H., et al., Oriented semiconducting polymer nanostructures as on-demand room-temperature single-photon sources. Applied Physics Letters, 2004. 85(1): p. 100-102.

39. Laurence, T.A., S. Fore, and T. Huser, Fast, flexible algorithm for calculating photon correlations. Optics Letters, 2006. 31(6): p. 829-831.

40. Walls, D. and G. Milburn, Quantum Optics. 1994, Berlin: Springer. 41. Moerner, W.E., R.M. Dickson, and D.J. Norris, Single-molecule nanophotonics in solids. Materials Science and Engineering B-Solid State Materials for Advanced Technology, 1997. 48(1-2): p. 169-174.

42. Berglund, A.J., A.C. Doherty, and H. Mabuchi, Photon statistics and dynamics of fluorescence resonance energy transfer. Physical Review Letters, 2002. 89(6): p. - .

43. Messin, G., et al., Bunching and antibunching in the fluorescence of semiconductor nanocrystals. Optics Letters, 2001. 26(23): p. 1891-1893.

44. Kitson, S.C., et al., Intensity fluctuation spectroscopy of small numbers of dye molecules in a microcavity. Physical Review A, 1998. 58(1): p. 620-627. 
45. Huston, A. and C. Reimann, Photochemical Bleaching of Adsorbed Rhodamine $6 \mathrm{~g}$ as a Probe of Binding Geometries on a Fused Silica Surface. Chemical Physics, 1991. 149(3): p. 401-407.

46. Nguyen, T.Q., V. Doan, and B.J. Schwartz, Conjugated polymer aggregates in solution: Control of interchain interactions. Journal of Chemical Physics, 1999. 110(8): p. 4068-4078.

47. Huser, T., M. Yan, and L.J. Rothberg, Single chain spectroscopy of conformational dependence of conjugated polymer photophysics. Proceedings of the National Academy of Sciences of the United States of America, 2000. 97(21): p. 11187-11191.

48. Huser, T. and M. Yan, Solvent-related conformational changes and aggregation of conjugated polymers studied by single molecule fluorescence spectroscopy. Journal of Photochemistry and Photobiology a-Chemistry, 2001. 144(1): p. 43-51.

Samantha Fore is a postdoctoral researcher at the University of California, Davis. She received the B.S. degree in physics from the University of Texas, Arlington, in 1999. She was a Student Employee Graduate Research Fellow with Lawrence Livermore National Laboratory (LLNL), Livermore, CA, and has conducted most of her Ph.D. dissertation research at LLNL through collaborations with the National Science Foundation Center for Biophotonics. Her research interests include single molecule spectroscopy of both fundamental biological systems.

Ted A. Laurence is a staff scientist at Lawrence Livermore National Laboratory. He received the B.S. degree from the California Institute of Technology, Pasadena, in 1977 and the Ph.D. degree from the University of California, Berkeley, in 2002. He was a National Science Foundation Graduate Fellow from 1997 to 2000. After postdoctoral research at University of California, Los Angeles, he became a Fellow in the Physical Biosciences Institute at Lawrence Livermore National Laboratory, Livermore, CA. His interests include biological applications of single molecule spectroscopy and surface-enhanced Raman spectroscopy.

Christopher Hollars is a senior scientist at the Midwest Research Institute in Kansas City, OK. He received his B.S. from the University of Central Oklahoma, Edmond, OK, in 1995 and the $\mathrm{Ph} . \mathrm{D}$. degree in chemistry from the University of Kansas, Lawrence, in 2000. His thesis and dissertation work involved the investigation of the submicron structure of model lipid membranes using near-field scanning optical microscopy combined with single molecule spectroscopy. He has been employed at Lawrence Livermore National Laboratory, Livermore, CA, since completing his graduate work as a Postdoctoral Associate from 2000 to 2001 and as a Staff Researcher from 2001 to 2007. His research interests include analytical method development using optical detection.

Thomas Huser an Associate Professor in the Department of Internal Medicine at the University of California, Davis. He is also Chief Scientist for the NSF Center for Biophotonics at UC Davis. Before coming to Davis, he was a Groupleader for Biophotonics and Nanospectroscopy at Lawrence Livermore National Laboratory in Livermore, CA. He obtained his Ph.D. in Physics from the University of Basel, Switzerland, where he worked primarily on near-field optical microscopy. He joined LLNL in 1998 as a postdoctoral associate and became a staff scientist in 2000. His research interest is in applications of single molecule fluorescence and single cell Raman spectroscopy to biological and medical problems. 